\title{
High Energy Astroparticle Physics
}

\author{
Günter Sigl ${ }^{\mathrm{a}}$ \\ ${ }^{a}$ APC (AstroParticules et Cosmologie), 10, rue Alice Domon et Lonie Duquet, 75205 Paris Cedex 13, \\ France and \\ Institut d'Astrophysique de Paris, 98bis Boulevard Arago, 75014 Paris, France
}

\begin{abstract}
We give a brief (and highly incomplete) overview of the current experimental and theoretical status of high energy cosmic rays and their secondary $\gamma$-rays and neutrinos. We focus on the role of large scale magnetic fields and on multi-messenger aspects linking these three channels. We also recall that the flavor composition of neutrino fluxes from astrophysical sources contains information on both the source conditions and neutrino physics.
\end{abstract}

\section{Introduction}

After almost 90 years of research, the origin of cosmic rays is still an open question, with a degree of uncertainty increasing with energy [1]. Above $\sim 100 \mathrm{MeV}$ the CR spectrum exhibits little structure and is approximated by broken power laws $\propto E^{-\gamma}$ : At the energy $E \simeq 4 \times 10^{15} \mathrm{eV}$ called the "knee", the flux of particles per area, time, solid angle, and energy steepens from a power law index $\gamma \simeq 2.7$ to one of index $\simeq 3.0$. The bulk of the CRs up to at least that energy is believed to originate within the Milky Way Galaxy, typically by shock acceleration in supernova remnants. These objects have been seen in $\gamma$-rays up to $\sim 10 \mathrm{TeV}$ by experiments such as HESS [2] and MAGIC [3], with a spectrum roughly scaling as $E^{-2.2}$. This is consistent with $\gamma$-ray production in interactions with the ambient gas of primary cosmic rays with a similar spectrum at the source. The difference to the cosmic ray spectrum observed to scale as $E^{-2.7}$ below the knee can be explained by diffusion in the galactic magnetic fields.

Above the knee the spectrum continues with a further steepening to $\gamma \simeq 3.3$ at $E \simeq 4 \times 10^{17} \mathrm{eV}$, sometimes called the "second knee". There are experimental indications that the chemical composition changes from light, mostly protons, at the knee to domination by iron and even heavier nuclei at the second knee [4]. This is in fact expected in any scenario where acceleration and propagation is due to magnetic fields whose ef- fects only depend on rigidity, the ratio of charge to rest mass, $Z / A$. This is true as long as energy losses and interaction effects, which in general depend on $Z$ and $A$ separately, are small, as is the case in the Galaxy, in contrast to extragalactic cosmic ray propagation at ultra-high energy. Above the so called "ankle" or "dip" at $E \simeq 5 \times 10^{18} \mathrm{eV}$, the spectrum flattens again to a power law of index $\gamma \simeq 2.8$. This latter feature is often interpreted as a cross over from a Galactic component, which steepens because cosmic rays are not confined by the galactic magnetic field any more or because Galactic sources do not accelerate beyond the ankle, to a harder component of extragalactic origin. However, the dip at $E \simeq 5 \times 10^{18} \mathrm{eV}$ could also be explained by pair production by extra-galactic protons, if the extragalactic component already starts to dominate below the ankle, for example, around the secondknee [5] at a few times $10^{17} \mathrm{eV}$. This requires a relatively steep injection spectrum $\propto E^{-2.6-2.7}$. Below a few times $10^{17} \mathrm{eV}$ this extra-galactic component would become unobservable at Earth due to diffusion in extra-galactic magnetic fields (EGMF) [6. In addition, the effective volumeaveraged injection spectrum has to become flatter somewhere below $\sim 10^{18} \mathrm{eV}$ in order to avoid excessive power going into cosmic rays and to avoid overproduction of $\mathrm{GeV}-\mathrm{TeV} \gamma$-rays from $p p$ interactions with the ambient gas.

The low cross-over scenario also requires the dominance of protons around the dip. Theoret- 
ically, this can be achieved either because preferentially protons are accelerated or because extended EGMF lead to strong photo-spallation during propagation 7]. Experimentally, above $\simeq 10^{17} \mathrm{eV}$ the chemical composition is basically unknown [8]. Around $10^{18} \mathrm{eV}$ the situation is particularly inconclusive as HiRes [9] and HiResMIA [10] data suggest a light (proton dominated) composition, whereas other experiments indicate a heavy composition [4. In any case, the cosmic ray flux should be extra-galactic at least above the ankle, where a galactic origin would predict an anisotropy toward the galactic plane because galactic magnetic fields can no longer isotropize the cosmic rays. No such anisotropy is seen. There are also experimental indications for a chemical composition becoming again lighter above the ankle, although a significant heavy component is not excluded and the inferred chemical composition above $\sim 10^{18} \mathrm{eV}$ is sensitive to the model of air shower interactions and consequently uncertain presently [8]. In addition, should a substantial heavy composition be experimentally observed up to the highest energies, some sources would have to be surprisingly nearby, within a few $\mathrm{Mpc}$, otherwise only low mass spallation products would survive propagation [11. In the following we will restrict our discussion on extra-galactic ultra-high energy cosmic rays (UHECRs).

No conclusive picture for the nature and distribution of the sources emerges yet naturally from the data [12]: Arrival directions are approximately isotropic [13], suggesting a large number of weak or distant sources. But there are also indications which point more towards a small number of local and therefore bright sources, especially at the highest energies: First, the AGASA ground array claimed statistically significant multi-plets of events from the same directions within a few degrees [14]13, although this is controversial [15] and has not been seen so far by other experiments such as the fluorescence experiment HiRes [16]. The spectrum of this clustered component is $\propto E^{-1.8}$ and thus much harder than the total spectrum [14]. Second, nucleons above $\simeq 70 \mathrm{EeV}$ suffer heavy energy losses due to photopion production on the cosmic microwave back- ground (CMB) - the Greisen-Zatsepin-Kuzmin (GZK) effect [17 — which limits the distance to possible sources to less than $\simeq 100 \mathrm{Mpc}$ [18]. This predicts a "GZK cutoff", a drop in the spectrum, whose strength depends on the source distribution and may even depend on the part of the sky one is looking at: The "cutoff" could be mitigated in the northern hemisphere where more nearby accelerators related to the local supercluster can be expected. Apart from the SUGAR array which was active from 1968 until 1979 in Australia, all UHECR detectors completed up to the present were situated in the northern hemisphere. Nevertheless the situation is unclear even there: Whereas a "cut-off" is consistent with the few events above $10^{20} \mathrm{eV}$ recorded by the fluorescence detector HiRes [19] and with the first data release of the Pierre Auger observatory [20, there is a tension with the 11 events above $10^{20} \mathrm{eV}$ detected by the AGASA ground array [21]. Still, this could be a combination of statistical and systematic effects 22, especially given the recent downward revision of the energy normalization in AGASA [23. The solution of this problem will have to await more analysis and more statistics and, in particular, the completion of the Pierre Auger project [20] which combines the two complementary detection techniques adopted by the aforementioned experiments and whose southern site is currently in construction in Argentina. Finally, about $1 \%$ of the HiRes steroa events around $10^{19} \mathrm{eV}$ seem to correlate with active galaxies of the BL Lac on a scale of $\sim 0.6^{\circ}$, with a significance of $\sim 10^{-4}$ 2425. Due to deflection of UHECR in the galactic magnetic field this, however, would have to be neutral primaries that cannot be created in the necessary quantities over the distances involved.

\section{Role of large scale magnetic fields}

The hunt for UHECR sources is further complicated by the presence of large scale cosmic magnetic fields which may significantly deflect charged cosmic rays even at the highest energies, in particular if sources correlate with high magnetic field regions such as galaxy clusters. A major issue in UHECR propagation studies 
is, therefore, the strength and distribution of EGMF. Galaxy clusters harbor magnetic fields of $\mu \mathrm{G}$ strength, but it is poorly known how quickly these fields fall off with increasing distance from the cluster center. The current data indicate that $\mu \mathrm{G}$ strength magnetic fields extend to at least $\sim 1$ Mpc [26] and possibly to larger distances [27/28]. Beyond $\simeq 1 \mathrm{Mpc}$ from a cluster core, however, probing the magnetic fields becomes extremely difficult because the Faraday Rotation Measure loses sensitivity in low density regions. Furthermore, the intracluster magnetic field topology is also poorly known, although the situation will likely improve in the future, for example with the advent of powerful radio astronomical instruments such as the square kilometer array.

One possibility in the meantime is to adopt large scale structure simulations (LSS) which include magnetic fields. In Ref. [29, the authors use magnetic fields derived from a cosmological LSS with magnetic fields generated at the shocks that form during LSS formation, whereas in Ref. 30. and Ref. 31 fields of "primordial" origin have been considered. While the different models for initial magnetic seed fields produce different large scale magnetic field distributions and, therefore, lead to different predictions for UHECR deflection, there is still a significant discrepancy between Ref. 29130 and Ref. 31, hinting that other technical reasons may play a role here. In the more extended fields from the simulations of Refs. 2910 deflection of protons up to $10^{20} \mathrm{eV}$ can be up to tens of degrees, whereas deflections in the simulations of Ref. 31] are typically below a degree. Assuming the EGMF correlates with the infrared luminosity density, Ref. 32 recently found results closer to Refs. 29130 than Ref. 31.

We recall that since acceleration is rigidity dependent, at the acceleration sites the highest energy cosmic ray flux is likely dominated by heavy nuclei. If this is indeed the case, it is interesting to point out that even in the EGMF scenario of Ref. [31, deflections could be considerable. In contrast to the contribution of our Galaxy to deflection which can be of comparable size but may be corrected for within sufficiently detailed models of the galactic field, the extra-galactic contribution would be stochastic. Statistical meth- ods are therefore likely to be necessary to learn about UHECR source distributions and characteristics as well as EGMF. For example, a suppressed UHECR arrival direction auto-correlation function at degree scales, rather than pointing to a high source density, could be a signature of extended EGMF 29.

Finally, EGMF can considerably increase the path-length of UHECR propagation and thus modify spectra, especially from individual sources, as well as the chemical composition observed at Earth [33].

\section{Multi-messenger approach: Secondary gamma-rays and neutrinos and their fla- vor composition}

The physics and astrophysics of UHECRs are also intimately linked with the emerging field of neutrino astronomy 34 as well as with the already well established field of $\gamma$-ray astronomy [35. Indeed, all scenarios of cosmic ray origin, from the galactic scale 36 to top-down 37 . and Z-burst models at the highest energies [40], are severely constrained by neutrino and $\gamma$-ray observations and limits. This has, for example, important consequences for theoretical predictions of diffuse fluxes of extragalactic neutrinos above about a $\mathrm{TeV}$ whose detection is a major goal of next-generation neutrino telescopes: If these neutrinos are produced as secondaries of protons accelerated in astrophysical sources and if these protons leave the sources and contribute to the UHECR flux observed, then the energy content in the neutrino flux can not be higher than the one in UHECRs, leading to the so called Waxman-Bahcall bound for transparent sources with soft acceleration spectra 4142. If one of these assumptions does not apply, such as for acceleration sources with injection spectra harder than $E^{-2}$ and/or opaque to nucleons, or if much fewer nucleons than $\gamma$-rays and neutrinos are produced, such as in top-down scenarios, the Waxman-Bahcall bound does not apply, but the neutrino flux is still constrained by the observed diffuse $\gamma$-ray flux in the $\mathrm{GeV}$ range.

Fig. 1 provides a sketch of "realistic" cosmic ray, $\gamma$-ray, and neutrino flux predictions in com- 


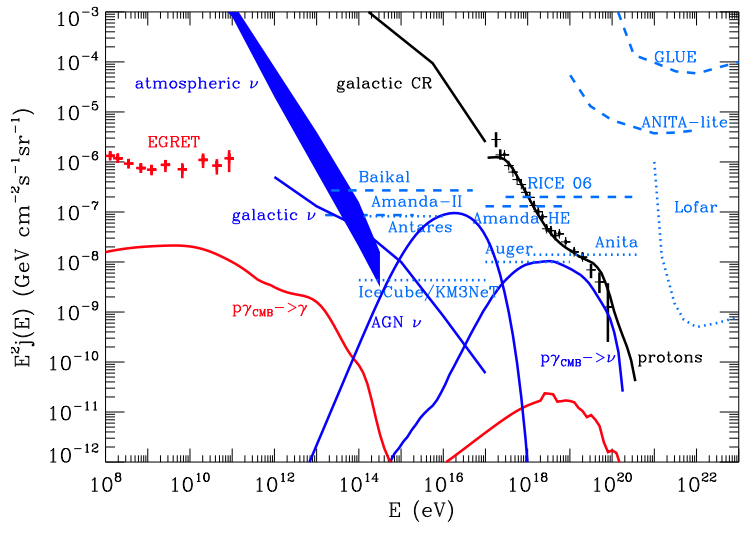

Figure 1. Model fluxes compared to experimental data, limits and sensitivities. Primary cosmic ray fluxes (data and a model, see text) are shown in black, the secondary $\gamma$-ray flux expected from proton interactions with the CMB and infrared background in red and the "guaranteed" neutrino fluxes per neutrino flavor in blue: atmospheric neutrinos, galactic neutrinos resulting from cosmic ray interactions with matter in our Galaxy [43, and "GZK" neutrinos resulting from cosmic ray interaction with the CMB and infrared background. The GZK neutrino fluxes depend on the distribution of the (unknown) primary cosmic ray sources for which we assumed active galactic nuclei (AGNs) above $10^{17} \mathrm{eV}$, using our public CRPropa code 38139. Cosmic ray interactions within these sources can also produce neutrinos for which one example is given $(\mathrm{AGN} \nu)$ [0]. The flux of atmospheric neutrinos has been measured by underground detectors and AMANDA. The dashed and dotted blue lines are existing upper limits and future sensitivities to diffuse neutrino fluxes from various experiments, respectively [34, assuming the Standard Model neutrino-nucleon cross section extrapolated to the relevant energies. The maximum possible neutrino flux would be given by horizontally extrapolating the diffuse $\gamma$-ray background observed by EGRET [4].

parison with experimental observations, limits, and sensitivities. It shows a theoretical scenario in which extra-galactic cosmic ray sources roughly evolving as quasars inject a spectrum $\propto E^{-2.6}$ of dominantly protons down to $\sim 10^{17} \mathrm{eV}$ where a cross-over to galactic cosmic rays occurs [5]. The "cosmogenic" neutrino flux produced by protons interacting with the low energy photon background considerably depends on these assumptions which can thus be used to test them [45].

Apart from cosmogenic neutrinos produced during propagation of UHECR, neutrinos can also be produced within astrophysical sources such as AGNs (see Fig. 1) or $\gamma$-ray bursts. In the absence of matter effects, a source at cosmological distances injecting neutrino fluxes with a flavor ratio $\propto w_{\beta}, \beta=e, \mu, \tau$, leads to a flavor mixture $\phi_{\alpha} \propto \sum_{\beta, i} w_{\beta}\left|U_{\alpha i}\right|^{2}\left|U_{\beta i}\right|^{2}$ observed at Earth, where $U_{\alpha i}$ is the mixing matrix and $i$ labels mass eigenstates. Therefore, if both pions and muons decay before loosing energy around the source, $w_{e}: w_{\mu}: w_{\tau} \simeq 1: 2: 0$ and thus $\phi_{e}: \phi_{\mu}: \phi_{\tau} \simeq 1: 1: 1$. At high energies the meson and muon energy loss time $t_{\text {loss }}(E)$ becomes shorter than their decay time $E \tau / m$, and the neutrino spectrum will be suppressed by a factor $\simeq m t_{\text {loss }}(E) /(\tau E)$ compared to primary interaction rates. For hadronic cooling, $t_{\text {loss }} \sim$ const, whereas for radiative cooling at the highest energies, $t_{\text {loss }}(E) \propto E^{-1}$, resulting in a steepening of the neutrino spectrum by a factor $E^{-1}$ and $E^{-2}$, respectively [46. In addition, at a given energy, charged pions decay about hundred times faster than muons. There can thus be an energy range at which pions but not muons decay before loosing energy such that $w_{e}: w_{\mu}: w_{\tau} \simeq 0: 1: 0$ and thus $\phi_{e}: \phi_{\mu}: \phi_{\tau} \simeq 1: 2: 2$. Also, $p p$ interactions produce both pions of both charges and thus give a higher fraction of $\overline{\nu_{e}}$ compared to $p \gamma$ interactions. The observed flavor ratios can thus depend on energy and carry information on the source conditions [47, but also about the mixing matrix itself [48].

Finally, flavor ratios can probe new physics, such as neutrino decay and quantum decoherence [49]: If all but the lightest mass eigenstate $j$ decay before reaching the observer, the flux of flavor $\alpha$ observed at Earth would be $\propto\left|U_{\alpha j}\right|^{2}$, independent of the flavor ratio at the source. For $j=1$ (normal mass hierarchy) this gives $\phi_{e}$ : 
$\phi_{\mu}: \phi_{\tau} \simeq 6: 1: 1$, whereas for $j=3$ (inverted mass hierarchy) one has $\phi_{e}: \phi_{\mu}: \phi_{\tau} \simeq 0: 1: 1$, which should be easy to distinguish from the normal case. This would allow to probe lifetimes of the order $\tau / m \sim 300(\mathrm{E} / \mathrm{PeV})^{-1} \mathrm{~s} / \mathrm{eV}$, which could improve on current limits. Quantum decoherence would predict $\phi_{e}: \phi_{\mu}: \phi_{\tau}=1: 1: 1$, independent of source flavor ratios.

Three-dimensional propagation in structured large scale magnetic fields also has considerable influence on secondary $\gamma$-ray and neutrino fluxes. Fig. 2 demonstrates how magnetic fields of $\mu \mathrm{G}$ strength surrounding a UHECR source, for example in a galaxy cluster, can influence the secondary GeV-TeV $\gamma$-ray fluxes produced by electromagnetic cascades initiated by UHECR interactions with the CMB and infrared background. This is the result of simulations with our public code CRPropa 38, discussed in Ref. 3950]. For the steep injection spectrum $\propto E^{-2.7}$ assumed in Fig. 2, the photon flux below a $\mathrm{TeV}$ is dominated by synchrotron radiation from electron/positron pairs produced by protons around the ankle. As a consequence, it depends considerably on strength and extension of EGMF around the source.

\section{REFERENCES}

1. for a recent introduction to cosmic rays see, e.g., T. Stanev, High Energy Cosmic Rays (Springer 2004).

2. F. Aharonian et al., Nature 432, 75 (2004).

3. N. Sidro [MAGIC Collaboration], arXiv:astro-ph/0610945.

4. J. R. Hoerandel, Astropart. Phys. 21, 241 (2004) arXiv:astro-ph/0402356.

5. R. Aloisio, V. Berezinsky, P. Blasi, A. Gazizov, S. Grigorieva and B. Hnatyk, arXiv:astro-ph/0608219; and refs therein.

6. M. Lemoine, Phys. Rev. D 71, 083007 (2005) arXiv:astro-ph/0411173.

7. G. Sigl and E. Armengaud, JCAP 0510, 016 (2005) arXiv:astro-ph/0507656.

8. A. A. Watson, Nucl. Phys. Proc. Suppl. 151, 83 (2006) arXiv:astro-ph/0410514.

9. R. U. Abbasi et al. [The High Resolution Fly's Eye Collaboration], Astrophys. J. 622, 910 (2005) arXiv:astro-ph/0407622.

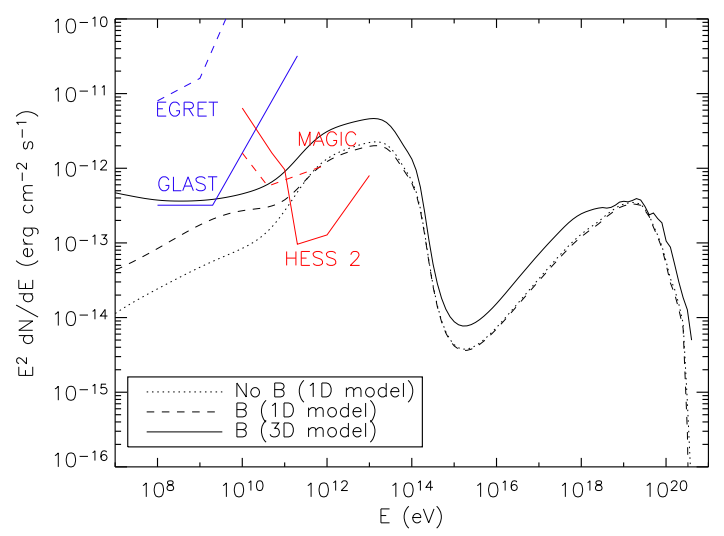

Figure 2. Differential $\gamma$-ray fluxes from electromagnetic cascades including synchrotron radiation, initiated by photo-pion and pair production by protons injected with an $E^{-2.7}$ spectrum (not shown) by a source at distance $d=20 \mathrm{Mpc}$, from Ref. [50]. We assume the source contributes a fraction $\simeq 0.2$ to the total UHECR flux, corresponding to a proton luminosity $\simeq 4 \times 10^{42} \mathrm{erg}$ $\mathrm{s}^{-1}$ above $10^{19} \mathrm{eV}$. A structured magnetic field of $0.1-1 \mu \mathrm{G}$ extends a few Mpc around the source in case of the $1 \mathrm{D}$ and $3 \mathrm{D}$ simulations which take into account synchrotron radiation of electrons and positrons. The 1D model neglects proton deflection whereas the 3D simulation follows 3dimensional proton trajectories. The latter case implies that the fluxes shown here would be extended over $\sim 5^{\circ}(20 \mathrm{Mpc} / d)$ on the sky. The fluxes are comparable to sensitivities of spacebased $\gamma$-ray (blue) and ground-based imaging air Čerenkov detectors (red).

10. T. Abu-Zayyad et al. [HiRes-MIA Collaboration], Astrophys. J. 557, 686 (2001) arXiv:astro-ph/0010652.

11. D. Harari, S. Mollerach and E. Roulet, arXiv:astro-ph/0609294.

12. for a recent review see, e.g., J. W. Cronin, Nucl. Phys. Proc. Suppl. 138, 465 (2005) arXiv:astro-ph/0402487.

13. W. S. Burgett and M. R. O'Malley, Phys. Rev. D 67, $092002 \quad$ (2003) arXiv:hep-ph/0301001. 
14. K. Shinozaki [AGASA Collaboration], Nucl. Phys. Proc. Suppl. 151, 3 (2006).

15. C. B. Finley and S. Westerhoff, Astropart. Phys. 21, 359 (2004) arXiv:astro-ph/0309159.

16. C. B. Finley et al., Proc. 28th International Cosmic Ray Conference, Tsukuba, Japan, 1 (2003) 433.

17. K. Greisen, Phys. Rev. Lett. 16, 748 (1966); G. T. Zatsepin and V. A. Kuzmin, JETP Lett. 4, 78 (1966) [Pisma Zh. Eksp. Teor. Fiz. 4, 114 (1966)].

18. F. W. Stecker, Phys. Rev. Lett. 21, 1016 (1968).

19. D. R. Bergman [HiRes Collaboration], arXiv:astro-ph/0609453

20. K. H. Kampert [Pierre Auger Collaboration], arXiv:astro-ph/0608136 see also http://www.auger.org.

21. M. Takeda et al., Phys. Rev. Lett. 81, 1163 (1998) arXiv:astro-ph/9807193; N. Hayashida et al., arXiv:astro-ph/0008102 see also http ://www-akeno.icrr.utokyo.ac.jp/AGASA/.

22. D. De Marco, P. Blasi and A. V. Olinto, Astropart. Phys. 20, 53 (2003) arXiv:astro-ph/0301497.

23. M. Teshima, Proceedings of CRIS 2006, Catania, Italy.

24. R. U. Abbasi et al. [HiRes Collaboration], Astrophys. J. 636, 680 (2006) arXiv:astro-ph/0507120.

25. D. S. Gorbunov, P. G. Tinyakov, I. I. Tkachev and S. V. Troitsky, JETP Lett. 80, 145 (2004) [Pisma Zh. Eksp. Teor. Fiz. 80, 167 (2004)] arXiv:astro-ph/0406654.

26. T. E. Clarke, P. P. Kronberg and H. Boehringer, Astrophys. J. 547, L111 (2001) arXiv:astro-ph/0011281].

27. M. Johnston-Hollitt and R. D. Ekers, arXiv:astro-ph/0411045.

28. F. Govoni, M. Murgia, L. Feretti, G. Giovannini, K. Dolag and G. B. Taylor, arXiv:astro-ph/0608433

29. G. Sigl, F. Miniati and T. A. Ensslin, Phys. Rev. D 70, 043007 (2004) arXiv:astro-ph/0401084.

30. G. Sigl, F. Miniati and T. Ensslin, Nucl.
Phys. Proc. Suppl. 136, 224 (2004) arXiv:astro-ph/0409098.

31. K. Dolag, D. Grasso, V. Springel and I. Tkachev, JCAP 0501, 009 (2005) arXiv:astro-ph/0410419.

32. A. Elyiv, arXiv:astro-ph/0611696

33. G. Sigl, JCAP 0408, $012 \quad$ (2004) arXiv:astro-ph/0405549; E. Armengaud, G. Sigl and F. Miniati, Phys. Rev. D 72, 043009 (2005) arXiv:astro-ph/0412525.

34. for recent reviews see, e.g., F. Halzen and D. Hooper, Rept. Prog. Phys. 65, 1025 (2002) arXiv:astro-ph/0204527; A. B. McDonald, C. Spiering, S. Schonert, E. T. Kearns and T. Kajita, Rev. Sci. Instrum. 75, 293 (2004) arXiv:astro-ph/0311343.

35. for a recent short review see, e.g., H. J. Völk, arXiv:astro-ph/0603501.

36. M. D. Kistler and J. F. Beacom, Phys. Rev. D 74, 063007 (2006) arXiv:astro-ph/0607082.

37. P. Bhattacharjee and G. Sigl, Phys. Rept. 327, 109 (2000) arXiv:astro-ph/9811011.

38. see http://apcauger.in2p3.fr//CRPropa.

39. E. Armengaud, G. Sigl, T. Beau and F. Miniati, arXiv:astro-ph/0603675.

40. D. V. Semikoz and G. Sigl, JCAP 0404, 003 (2004) arXiv:hep-ph/0309328.

41. E. Waxman and J. N. Bahcall, Phys. Rev. D 59, 023002 (1999) arXiv:hep-ph/9807282; J. N. Bahcall and E. Waxman, Phys. Rev. D 64, 023002 (2001) arXiv:hep-ph/9902383.

42. J. P. Rachen, R. J. Protheroe and K. Mannheim, arXiv:astro-ph/9908031.

43. J. Candia and E. Roulet, JCAP 0309, 005 (2003) arXiv:astro-ph/0306632.

44. A. W. Strong, I. V. Moskalenko and O. Reimer, arXiv:astro-ph/0306345.

45. see, e.g., T. Stanev, arXiv:astro-ph/0607515.

46. S. Ando and J. F. Beacom, Phys. Rev. Lett. 95, 061103 (2005) arXiv:astro-ph/0502521.

47. T. Kashti and E. Waxman, Phys. Rev. Lett. 95, 181101 (2005) arXiv:astro-ph/0507599.

48. P. D. Serpico, Phys. Rev. D 73, 047301 (2006) arXiv:hep-ph/0511313.

49. D. Hooper, Czech. J. Phys. 56, A337 (2006) arXiv:hep-ph/0510097.

50. E. Armengaud, G. Sigl and F. Miniati, Phys. Rev. D 73, 083008 (2006). 